Bull. Geol. Soc. Finland 41, 235-251 (1969)

\title{
ÜBER FINNLANDS REZENTE UND SUBFOSSILE DIATOMEEN III
}

\author{
KaRL MöLder und Risto Tynni \\ Geologische Forschungsanstalt in Finnland, Otaniemi, Finnland
}

\begin{abstract}
The article deals with the distribution and ecology of the Tetracyclus, Rhabdonema, Tabellaria, Grammatophora, Licmophora, Meridion, Diatoma, Plagiogramma, Dimerogramma and Opephora species known in Finland.
\end{abstract}

\section{INHALT}

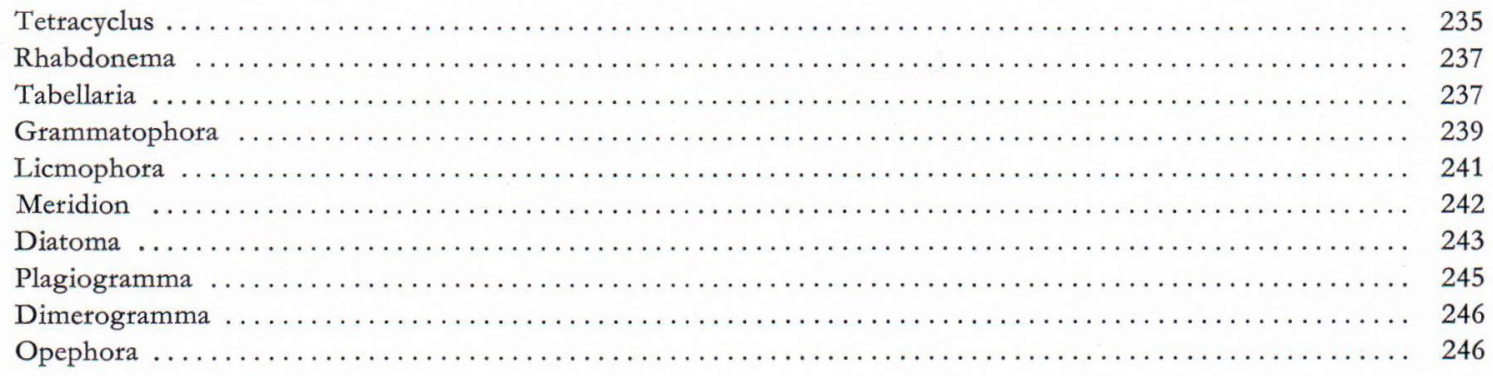

\section{Tetracyclus}

Die Schalen sind länglich, in ihrem mittleren Teil transapikal verbreitert oder oval geformt. Zuweilen ist die Verbreitung der mittleren Partie zweiteilig (T. emarginatus). In den Schalen zeigt sich eine apikal gerichtete Pseudoraphe, \pm rechtwinklig zu ihr unterscheiden sich die Rippen und in dichteren Abständen die Transapikalstreifen. Die Gürtelbandseite ist rechtwinklig, und in ihr lassen sich die Zwischenbänder mit ihren kurzen Septen abwechselnd an den apikalen Enden der parallelen Zwischenbänder erkennen.

\section{Tetracyclus emarginatus (Ehr.) W. Smith}

Synonyme: Biblarium emarginatum Ehr., Tetracyclus lacustris var. emarginata Cleve, Tetracyclus emarginatus $f$. abnormis Fontell.

Seltene Litoralform von Seen und Weihern. Kaltwasserform, deren Vorkommensdichte in Lappland grösser ist als in Südfinnland. Abb. 1. Die subfossilen Funde in Seesedimenten verhältmässig häufig.

Tetracyclus lacustris Ralfs

Synonyme: Biblarium stella Ehr., Tetracyclus stella Hèrib., Tetracyclus lacustris var. fossilis Pantocsek. 


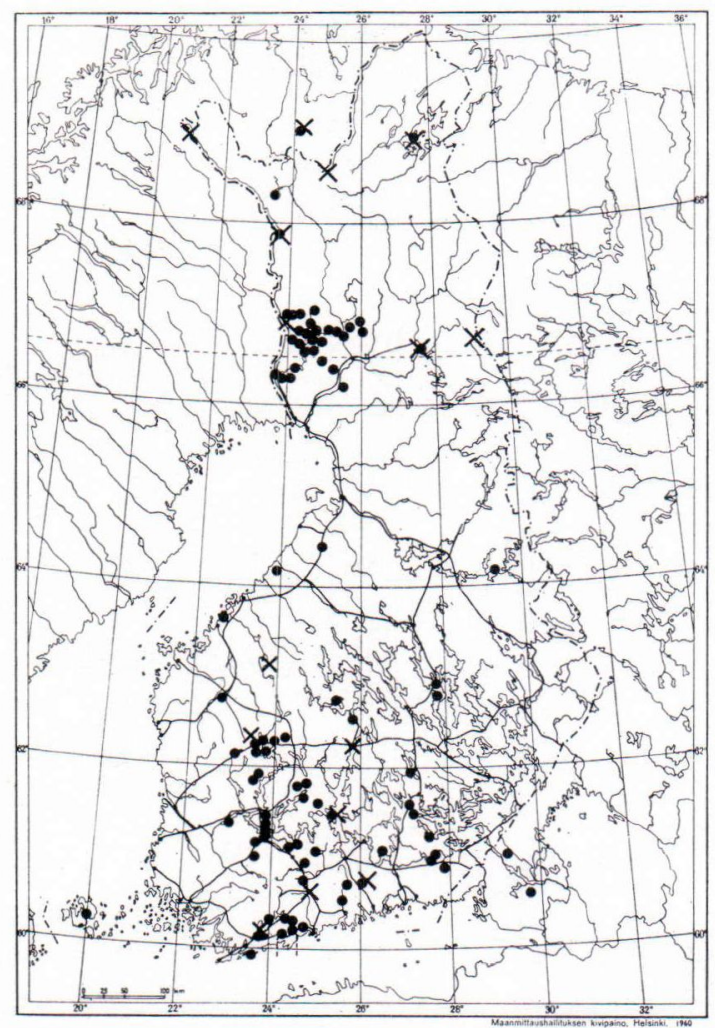

Abb. 1. Rezente Verbreitung von Tetracyclus emarginatus (Kreuze) und Tetracyclus lacustris (Punkte).

Litoralform von Seen und Flüssen, häufiger als die vorgenannte. Wächst auch auf Moosen. Ebenfalls Kaltwasserform, aber nicht in so grossem Masse wie die vorige Art. Wahrscheinlich acidophil, da sich die Beobachtungen auf ein dystrophes Seetypgebiet (Järnefelt 1935) konzentrieren. Abb. 1.

\section{Forma maxima Cleve-Euler}

Synonym: Tetracyclus elongata Hustedt.

Im Flusse Muonionjoki, im See Kemijärvi (Cleve-Euler 1953) und im See Järvelän Isojärvi (Siuntio) beobachtet (Järnefelt 1925).

Var. capitata Hustedt

Nur im See Lankojärvi in Pello aufgefunden, aber in Seesedimenten stellenweise relativ häufig,

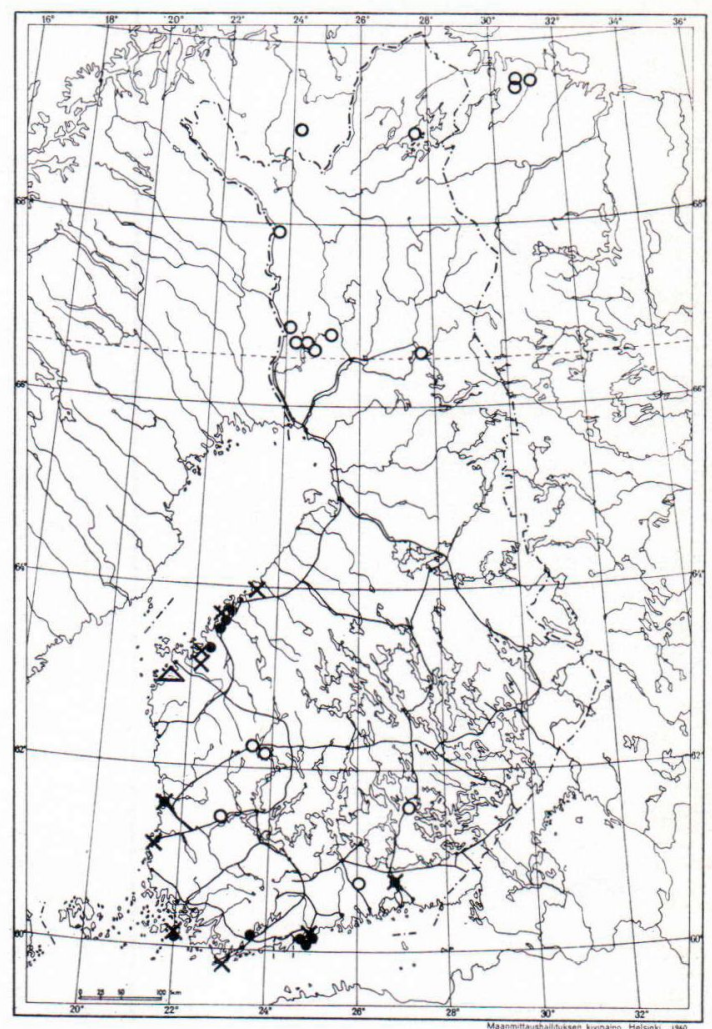

Abb. 2. Rezente Verbreitung von Tetracyclus lacustris var strumosa (Ringe), Rhabdonema arcuatum (Punkte), Rhabdonema minutum (Kreuze) und Rhabdonema torelli (Dreieck).

mit der Hauptform eine lückenlose übergansreiche bildend.

Var. elegans (Ehr.) Hustedt

Synonyme: Biblarium elegans Ehr., Tetracyclus elegans Ralfs.

Nur auf Aland rezent beobachtete Form (Mölder 1946 b).

Var. strumosa (Ehr.) Hustedt

Synonyme: Biblarium strumosum Ehr., Tetracyclus lacustris var. rhombica Hustedt, Schmidt's Atlas Taf. 269.

Mit der Hauptform oft vorkommende Kaltwasserform. Abb. 2. 
Tetracyclus rupestris (A. Braun) Grunow

Synonym: Tetracyclus braunii Grun.

In Südfinnland im See Kalljärvi und in der Pojo-Bucht sehr selten angetroffen. Nach Hustedt (1931) wächst die Art besonders auf Moos, sogar auf Kalkfels in Niederösterreich.

\section{Rhabdonema}

Die Zellen bestehen aus länglichen an ihren Enden abgerundeten Valvarflächen und rechteckigen Gürtelbändern. In der Valvarfläche zeigen sich eine Pseudoraphe und rechtwinklich gegen sie gerichtete, aus Punkten bestehende Transapikalstreifen sowie zuweilen im Randteil schwache Rippen. Das Zwischenband bildet in den Schalen eine die ovale Öffnung umgebende Wandung. In der Gürtelbandseite unterscheiden sich viele Zwishenbänder in ihren beiden apikalen Enden mit tief in den Innenteil reichenden Septen.

\section{Rhabdonema arcuatum (Ag.) Kütz.}

Synonyme: Diatoma arcuatum Lyngbye, Rhabdonema crozierii Grun., Rhabdonema ehrenbergii Fricke, Rhabdonema arcuatum var. maxima $\mathrm{A}$. Cleve.

An der Küste des Finnischen und Bottnischen Meerbusens wachsende litorale Epiphytform. Abb. 2. Subfossil häufig in marinen Litorinaund Postlitorina-Ablagerungen. Erscheint auch in yoldiazeitlichen und älteren marinen Ostseesedimenten.

Forma contracta Fricke

Rezent nur in der Pojo-Bucht, in Litorinaund Postlitorina-schichten neben der Hauptform selten angetroffen.

\section{Rhabdonema minutum Kützing}

Synonyme: Fragilaria striatula Greville, Tessella catena Ralfs.

In der Litoralzone des Finnischen und des Bottnischen Meerbusens in Brackwasser wach- sende Art, obschon seltener als $R h$. arcuatum. Abb. 2. Subfossil vorwiegend in marinen Litorina- und Postlitorina-Ablagerungen.

Rhabdonema torelli Cleve

Besonders eine Litoralform des Nördlichen Eismeeres (Hustedt 1931). Nur einmal an der Küste des Bottnischen Meerbusens in Åminne in Maalahti angetroffen. Abb. 2.

\section{Tabellaria}

Die Schalen länglich, bei den meisten Formen linear sowie im mittleren Teil und an den Enden aufgetrieben. Tabellaria binalis ist ausnahmsweise im mittleren Teil schmäler eingeschnürt. Die Pseudoraphe zwischen den Transapikalstreifen ist schmal, Rippen fehlen. Zwischenband an dem einen Ende offen und an dem anderen ein Septum, das bei den übrigen Arten, ausser bei T. binalis, bis nahe an die Mitte heran reicht. $T$. flocculosa hat bisweilen ausser dem Septum am entgegengesetzten Ende auch ein rudimentäres Septum (Knutson 1952). An der Gürtelbandseite sind 4 ( $T$. fenestrata + var. asterionelloides) oder mehr Zwischenbänder und Septen zu erkennen. Die Zellen sind zu gleichgerichteten ( $T$. fenestrata), über Eck zickzackförmigen ( $T$. fenestrata var. intermedia, $T$. flocculosa) oder sternförmigen (T. fenestrata var. asterionelloides) Kolonien aneinander befestigt. Die Art und Weise der Zellen, sich zu Kolonien gruppieren, ist im allgemeinen im subfossilen Material nicht zu erkennen. Die beigefügte Taxonomie richtet sich nach Hustedt (1931) und unterscheidet sich in gewissen Hinsichten u.a. von der von Cleve-Euler (1953) und Knutson $(1952,1953,1954)$ angewandten.

\section{Tabellaria binalis (Ehr.) Grunow}

\section{Synonyme: Fragilaria? binalis Ehr., Tetracyclus lewisianus} Östrup.

Die seltenste Tabellaria-Art, die in einigen nährstoffarmen Seen mit niedrigem $\mathrm{pH}$-Wert wächst. Nach Hustedt (1931) kommt die Art in Nord- und Mitteleuropa selten vor. Fundorte: 


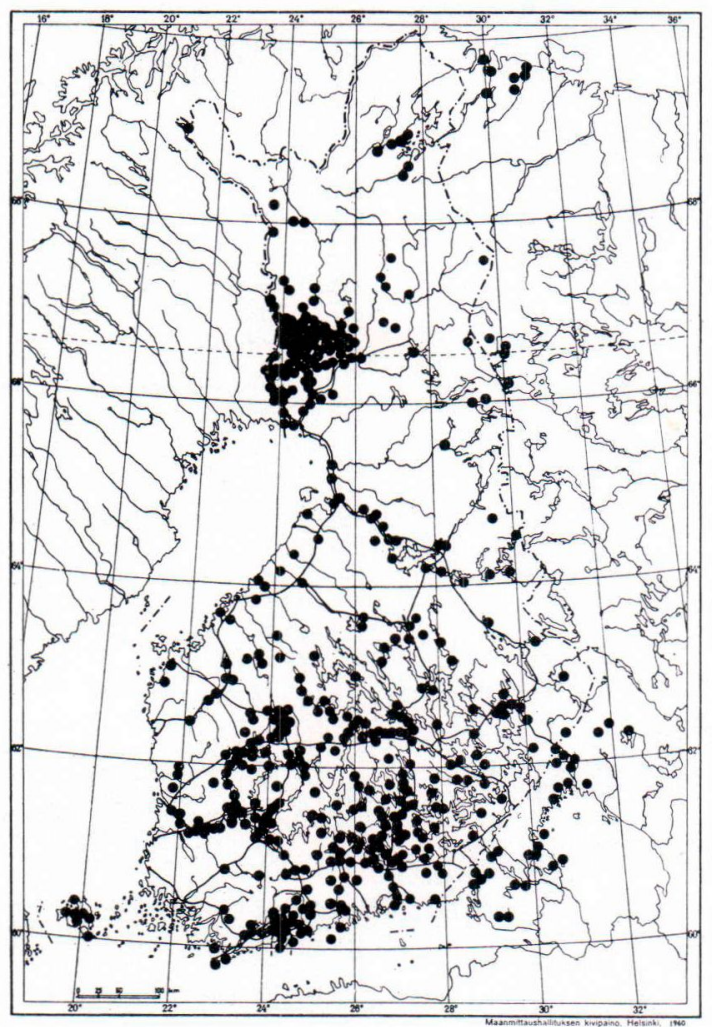

Abb. 3. Rezente Verbreitung von Tabellaria fenestrata.

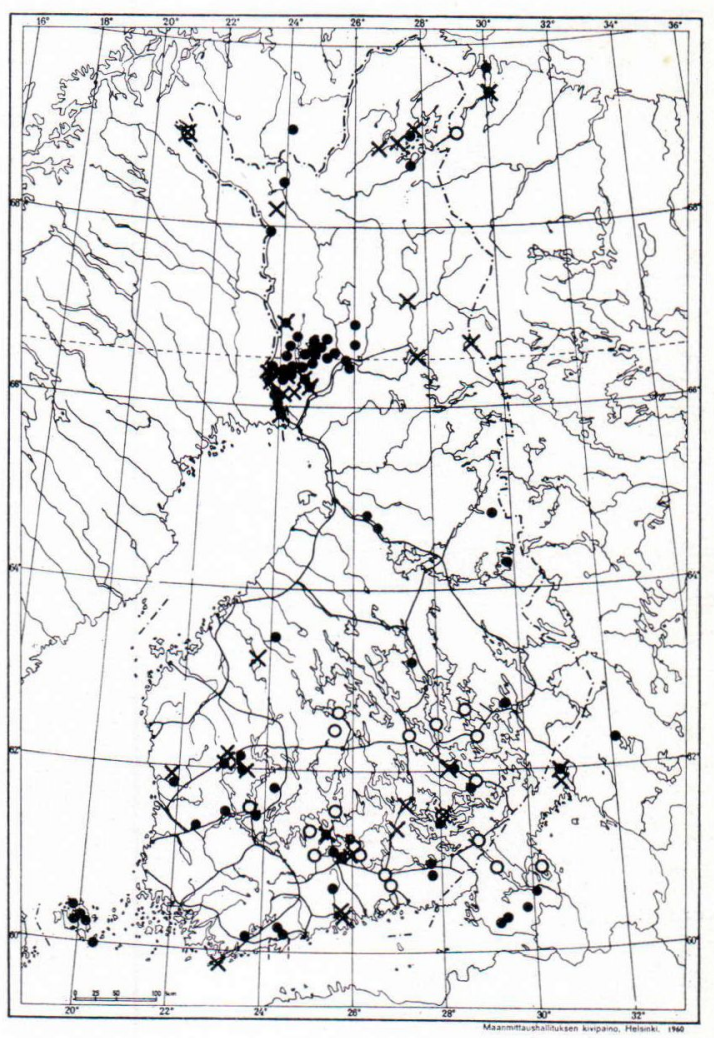

Abb. 4. Rezente Verbreitung von Tabellaria fenestrata var asterionelloides Punkte, T.f. var. geniculata (Ringe) und T.f. var. intermedia (Kreuze).

anderem nach Hustedt (1957) und Foged (1964) ist die Art acidophil (Hustedt 1938/39 S. 284). Nach Välikangas (1926) und Mölder (Halme \& Mölder 1958) ist die Art oligohalin und wächst auch in schwach brackigem Wasser an der Küste. Gut im Plankton bleibend, gerät die Art mit den Flüssen in das Küstengebiet. Im Brackwasser in der Pojo-Bucht in der Sommerzeit allgemein angetroffen. Dem Vorkommen und der Individuenzahl der Art in finnischen Seen hat Järnefelt in manchem Zusammenhang Aufmerksamkeit zugewandt (u.a. Järnefelt 1956). Abb. 3. Ausser in Süsswassersedimenten findet sich die Art oft in Ostseesedimenten, wenn auch in geringer Menge.

\section{Var. asterionelloides Grunow}

Synonyme: Tabellaria fenestrata var. willei Huitfeldt- 
Kaas, Tabellaria fenestrata var. lacustris Meister, Tabellaria fenestrata var. lacustris $\mathrm{f}$. asterionelloides Grunow.

Durch ihre Kolonienbildung von der Hauptform unterschieden. Eine in Finnland verhältnismässig häufige Form (Abb. 4), aber in subfossilem Material aus dem Kolonieverband zerstreut, sie kann von der Hauptform nicht unterschieden werden. Doch sind die ökologischen Ansprüche bei beiden gleich.

Var. genigulata A. Cleve

Verhältnismässig seltene Variation, die in erster Linie mit oligotrophen, relativ kalten Binnengewässern zusammenzuhängen scheint. (Abb. 4.) Levander und Wuorentaus (1915, 1917) haben u.a. bei ihren Untersuchungen diese Art beobachtet.

Var. intermedia Grunow

Eine in ähnlichen Gewässern wie die Hauptform wachsende Variation. (Abb. 4.) Nach vielen Forschern bildet diese eine Zwischenform nach Tabellaria flocculosa hinüber.

\section{Tabellaria flocculosa (Roth) Kützing}

Synonyme: Conferva flocculosa Roth, Tabellaria flocculosa var. ampbicephala Grun., Tabellaria flocculosa var. ventricosa (Kütz.) Grun., Tabellaria flocculosa var. genuina Kirchner.

Eine der häufigsten Süsswasserdiatomeen Finnlands. Die vielleicht häufigste Diatomee im subarktischen Gebiet, deren optimale Verhältnisse in bezug auf die Temperatur und auf das $\mathrm{pH}$ des Wassers in Schwedisch-Lappland 5.5-15. $5^{\circ} \mathrm{C}$ und $\mathrm{pH}$ 6-7 sind (Hustedt 1942). Die Art wächst in Seen, Weihern, Flüssen und Bächen unterschiedlichen Trophiegrades, aber ihre Abundanz ist in sauren Gewässern am grössten (vgl. Krasske 1949). Nach Round (1960) gehört die Art zu oligotroph bis dystrophen Gewässern. Gemäss Välikangas (1926) und Mölder (Halme \& Mölder 1958) ist sie eine oligohaline Form. Nach Mölder (1951) T. flocculosa-Formen, die in der Nähe des Eisrandes wachsen, sind besonders klein.

Hauptsächlich eine Litoralform, die subfossil vorzugsweise in Sedimenten kleiner Weiher häufig vorkommt. Abb. 5.

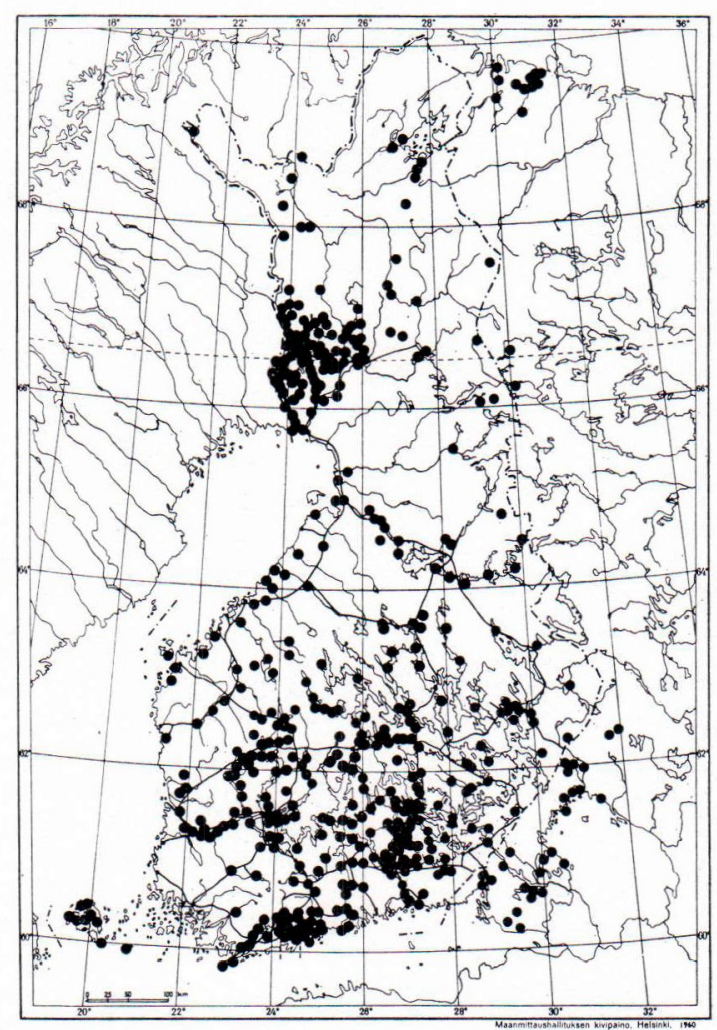

Abb. 5. Rezente Verbreitung von Tabellaria flocculosa.

\section{Grammatophora}

Die Valvarebenen linear länglich, selten bogig gekrümmt ( $G$. arcuata). Pseudoraphe schmal, unterbricht in der Membran die transapikale Punktliniierung, die sich bei den meisten Formen ausserdem auch entweder in apikal gerichteten Reihen (G. angulosa, G. arctica, G. arcuata) oder schräge in zwei einander rechtwinklig schneidenden Reihen angeordnet hat. Das Zwischenband bildet in der Valvarebene eine Wandung rings um die Öffnung, und ausserdem gestalten sich in der Valvarfläche näher an die Apikalenden heran andere Öffnungsfiguren. An der Gürtelbandseite zeigt sich bei ruhenden Zellen ein Zwischenband mit Septen und kleinen Pseudosepten in jeder Zellenhälfte.

Grammotophora angulosa Ehr. 


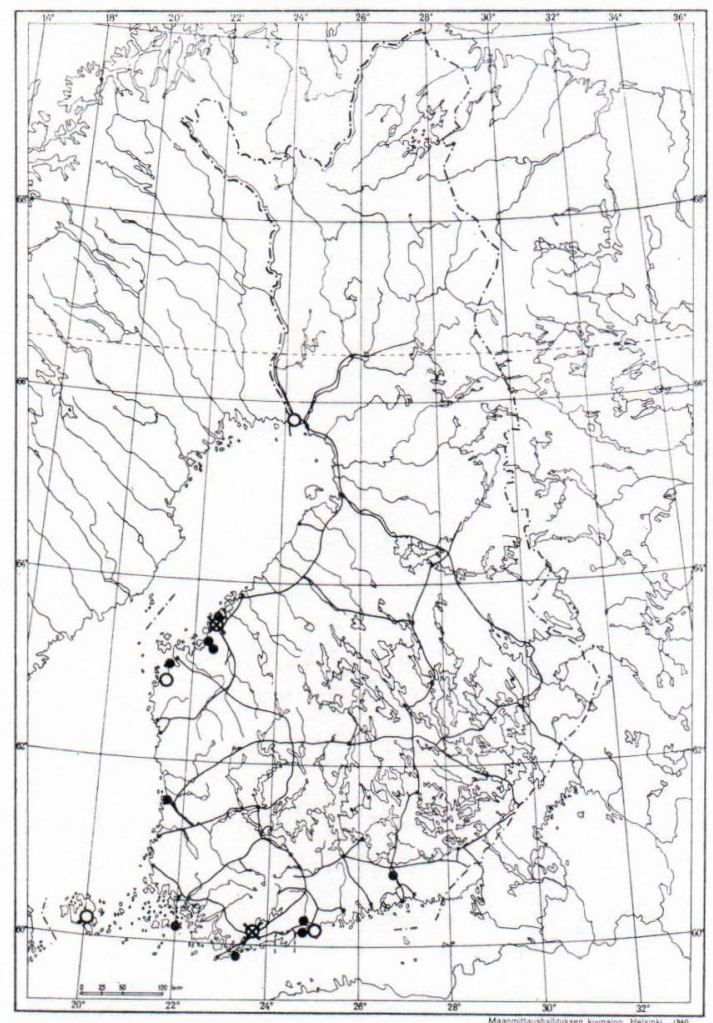

Abb. 6. Rezente Verbreitung von Grammotophora marina (Ringe), Grammotophora oceanica (Punkte) und G.o. var. macilenta (Kreuze).

Salzwasser-Litoralform, die an der Küste des Finnischen Meerbusens, bei Helsinki, nur vereinzelt angetroffen worden ist, wie auch var. islandica (Ehr.) Grun. Die Hauptform ist u.a. im Interglazialton von Rouhiala gefunden worden (Brander 1941).

\section{Grammotophora arcuata Ehr.}

Eine Form des arktischen oder antarktischen Meeres, die u.a. überall an den Küsten des nördlichen Eismeeres wächst (Hustedt 1931). In Finnland nur subfossil im interglazialen Ton von Rouhiala sowie in der Interglazialablagerung von Somero (Gardemeister 1968) angetroffen. In Schweden erscheint die Art in der spätglazialen Sedimentreihe im Tal des Göta-Flusses am häufigsten an der Ablagerungsstelle, deren Entstehung klimatisch den Verhältnissen der Jüngeren Dryaszeit entspricht (Miller 1964).

\section{Grammotophora marina (Lyngbye) Kützing}

Synonyme: Diatoma marinum Lyngbye, Grammatophora mexicana Ehr., Grammatophora marina var. maior Grun., Grammatophora marina var. lyngbyei Grun., Grammatophora marina var. mexicana Grun.

Salzwasserform, die auch im Brackwasser im Küstengebiet Finnlands wächst. (Abb. 6.) Häufig in Litorina- und Postlitorinasedimenten, dagegen in Yoldiasedimenten vergleichsweise selten. Unter anderem in marinen Interglazialsedimenten in Rouhiala, Somero (Gardemeister, op. cit.) und im Mga-Flusstal aufgefunden (Zans 1936).

\section{Var. adriatica Grunow}

Synonym: Grammatophora oceanica var. adriatica (Grun.) A. $\mathrm{Cl}$.

Salzwasserform, nur in der Pojo-Bucht angetroffen.

\section{Grammotophora oceanica (Ehr.) Grunow}

Synonyme: Grammatophora marina var. communis V. Heurck, Grammatophora oceanica var. communis (Grun.) A. Cl.,

Im Küstengebiet Finnlands verhältnismässig häufige Salzwasserform. (Abb. 6.) Die im Flusse Kymijoki und in Ostbottnien gemachten Funde sind wahrscheinlich aus marinen Sedimenten ausgewachsen. Obgleich die Art eigentlich eine Litoralform ist, hält sie sich gut im Plankton, kommt u.a. in der freien Wasserfläche des Bottnischen Meerbusens vor und sedimentiert sich auch fern vom Ufer im Tiefwassergebiet.

Häufig in Litorina- und Postlitorina-Ablagerungen, stellenweise auch in Yoldiaschichten. Erscheint in marinen Spätglazial- und Interglazialsedimenten.

\section{Var. macilenta (W. Sm.) Grunow}

Synonyme: Grammatophora macilenta W. Smith, Grammatophora oceanica var. subtilis Grun., Grammatophora marina var. macilenta V. Heurck., Grammatophora macilenta var. nodulosa Grun., Grammatophora oceanica var. macilenta f. nodulosa (Grun.) Hust., Grammatophora oceanica var macilenta f. vulgaris (Grun.) Hust. 
Salzwasserform, an der finnischen Küste in der Pojo-Bucht und vor Uusikaarlepyy im Bottnischen Meerbusen angetroffen. (Abb. 6). Subfossil in den gleichen Sedimenten wie die Hauptform, wenn auch selten, aufgefunden.

\section{Licmophora}

Die Valvarseite kegel- oder keulenförmig, die Pseudoraphe schmal sowie die Transapikalstreifen im allgemeinen zarter oder gröber punktiert, zuweilen statt ihrer Transapikalrippen (L. ehrenbergii). Bei Licmophora juergensi liegt die Punktierung ebenfalls in apikalgerichteten Reihen. Die weite Öffnung, wie die Kontur der Valvarfläche geformt, ist in jeder ruhenden Zellenhälfte vom Zwischenband breiter umrandet am oberen Ende, wo auch ein Septum zu sehen ist. Gürtelbandseite kegelförmig. Alle Arten sind marin, beim Wachsen vom Fusspol aus durch einem Gallertstrang an der Unterlage befestigt.

\section{Licmophora debilis (Kütz.) Grunow}

Synonyme: Podosphenia debilis Kützing, Licmophora debilis var. laevissima Grun., Licmophora debilis f. elongata V. Heurck.

Kleine Salzwasserform, nur bei Tvärminne angetroffen.

\section{Licmophora ebrenbergii (Kütz.) Grunow}

Synonyme: Podosphenia ebrenbergii Kützing.

Im Küstengebiet Finnlands nur in Tvärminne gefunden. Wahrscheinlich ein zufälliges Vorkommen.

\section{Forma angustata Grunow}

Synonyme: Podosphenia angustata Grun., Licmophora grunowii var. elongata Mereschkowsky.

Nur auf Raippaluoto erbeutete, wahrscheinlich nur zufällig im Bottnischen Meerbusen vorkommende Diatomee,

Forma elegans (Mereschkowsky) Hustedt

Nur subfossil in Tvärminne aufgefunden.
Licmophora gracilis (Ehr.) Grun.

Synonym: Podosphenia gracilis Ehr.

In der Pojo-Bucht und westlich von Helsinki gefundene Art. Nach Cleve-Euler (1953) anscheinend in der Ostsee allgemein verbreitete Art.

Var. anglica (Kütz.) Peragallo

Synonyme: Podosphenia gracilis var. minor Kütz., Licmophora anglica f. elongata Grun.

Neben der Hauptform im Küstengebiet der Ostsee wachsende Art.

\section{Licmophora juergensii Agardh}

Synonyme: Podosphenia juergensii Kützing. Licmophora juergensii var, intermedia Grunow.

Nur westlich von Helsinki angetroffene Art (Mölder \& Tynni 1966).

\section{Licmophora nubecula (Kütz.) Grunow}

Synonym: Licmophora tincta var. nubecula (Kütz.) Grunow.

Im J. 1938 im Gebiet Petsamo am Ufer der Insel Iso-Heinäsaari aufgefunden.

\section{Licmophora oedipus (Kütz.) Grunow}

Synonyme: Rhipidophora oedipus Kütz., Licmophora oedipus f. elongata Grun., Licmophora juergensii var. oedipus Peragallo.

In Korsnäs, der Pojo-Bucht, auf Åland und westlich von Helsinki angetroffene seltene Art. (Abb. 7.) Subfossil u.a. im Interglazialton von Rouhiala (Brander 1937) aufgefunden.

Licmophora paradoxa (Lyngbye) Agardh

Synonyme: Ecbinella paradoxa Lyngbye, Licmophora paradoxa var. australis De Toni.

Eine im Küstengebiet des Finnischen Meerbusens seltene Art, die in der Pojo-Bucht auf Järnö und bei Tvärminne sowie in Lappohja angetroffen worden ist. (Abb. 7). 


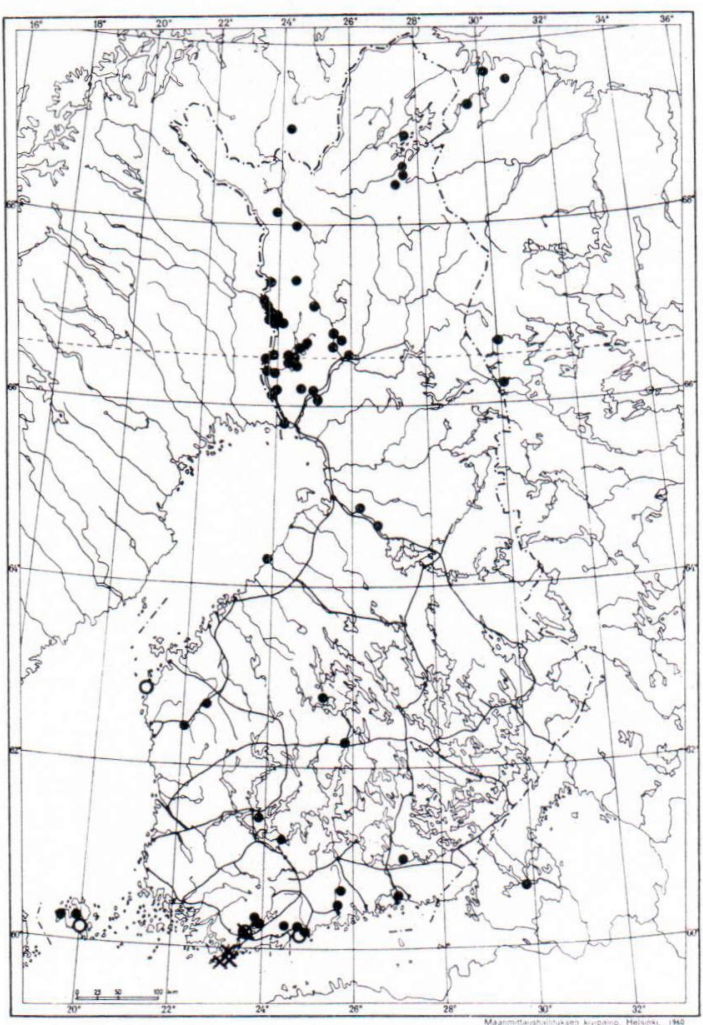

Abb. 7. Rezente Verbreitung von Licmophora oedipus (Ringe), Licmophora paradoxa (Kreutze) und Meridion circulare (Punkte).

\section{Meridion}

Die Valvarfläche mit keulenförmiger Kontur oder am oberen Pol durch Einschnürung kopfig (M. circulare var. constricta). Pseudoraphe schmal. Neben Transapikalliniierung in lichterem Abstand Rippen. An den Gürtelbandseiten sind Zwischenbänder zu unterscheiden, aber Septen fehlen. Die Gürtelbandseite ist kegelförmig, wodurch das Aneinanderfügen mehrerer Zellen einen fächerförmigen Komplex bildet.

\section{Meridion circulare (Grev.) Agardh}

Synonyme: Ecbinella circularis Greville, Meridion flabellum Ehr., Meridion circulare var. genuina Kirchner, Meridion circulare var. maxima von Alten, Meridion circulare var. genuina f. gracilis A. Mayer Meridion béribaudi $\mathbf{M}$. Peragallo, Meridion circulare f. ovata A. Cl.

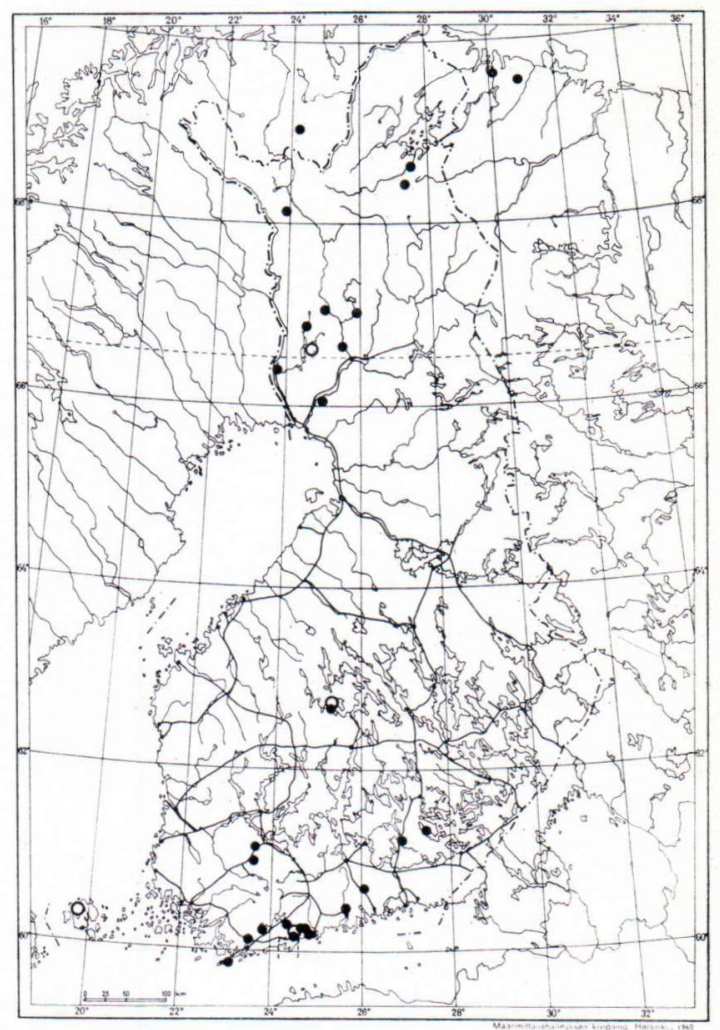

Abb. 8. Rezente Verbreitung von Meridion circulare var. constricta (Punkte) und Diatoma anceps (Ringe).

Eine vorwiegend in Bächen und Quellen zeitweilig häufige Kaltwasserform. Im Frühjahr zur Zeit der Schneeschmelze bildet die Art oft Massenvorkommen, wandert mit kleinen Bächen in Seen und gelangt in Sedimente. Nach CleveEuler (1953) und Niessen (1956) eine »kalziphile», nach Foged (1964) eine alkaliphile Form. Abb. 7.

Var. constricta (Ralfs) van Heurck

Synonyme: Meridion constrictum Ralfs.

Eine neben der Hauptform in gleichartigen Gewässern vorkommende Variatio (Abb. 8). Oft häufiger als jene. 


\section{Diatoma}

Die Valvarflächen linear bis elliptisch geformt, zuweilen in der Mitte eingeschnürt ( $D$. vulgare var. constricta). Bei bestimmten Formen sind die apikalen Enden kopfig, entweder durch Anschwellen der Pole (D. elongatum) oder durch Einschnüren der Ränder (D. vulgare var. capitulata und var. grandis, $D$. anceps). Die Pseudoraphe im allgemeinen deutlich zu unterscheiden. Die punktierten Transapikalstreifen sind meistens dünn. Die Rippen liegen bei $D$. vulgare + var. und $D$. elongatum + var. verhältnismässig nahe beieinander, bei den übrigen lichter. An dem einen Ende der Valvarebene zeichnet sich eine Gallertpore ab. An der Gürtelbandseite lassen sich bei den meisten Arten Zwischenbänder erkennen, die bei $D$. biemale am zahlreichsten sind (4-5). Septen fehlen. Im Plankton am häufigsten sind die Formen, die in zickzack- oder sternförmigen Kolonien wachsen, in fliessendem Wasser wiederum diejenigen, die nebeneinander geschlossene Kolonien bilden.

Diatoma anceps (Ehr.) Kirchner

Synonyme: Odontidium anceps Ehr., Diatoma anceps var. anomala, var. capitata, var. constricta, var. linearis Tampère \& Peragallo, Diatoma biemale var. anceps (Ehr.) A. Cl.

Rezent selten angetroffene Art. (Abb. 8). Nach Hustedt wachst die Art vornehmlich im fliessenden Wasser von Gebirgen und deren Vorland.

\section{Diatoma elongatum (Lyngb.) Agardh}

Synonyme: Diatoma tenue var. elongatum Lyngbye.

Sie ist die in Finnland häufigste Diatoma-Art, die vorwiegend in schwach salzhaltigem, kühlem Brackwasser an der Küste wächst (Välikangas 1926: mesohalines Brackwasser), aber auch im Binnenland in Gewässern unterschiedlichen Trophiegrades, u.a. in Grossseen. (Abb. 9.).

Nach Krasske (1938) und Scheele (1952) ist die Art halophil. In bezug auf den $\mathrm{pH}$-Wert des Wassers ist die Art indifferent (Hustedt 1957). Planktonform. Häufig in Süss- und BrackwasserSedimenten.

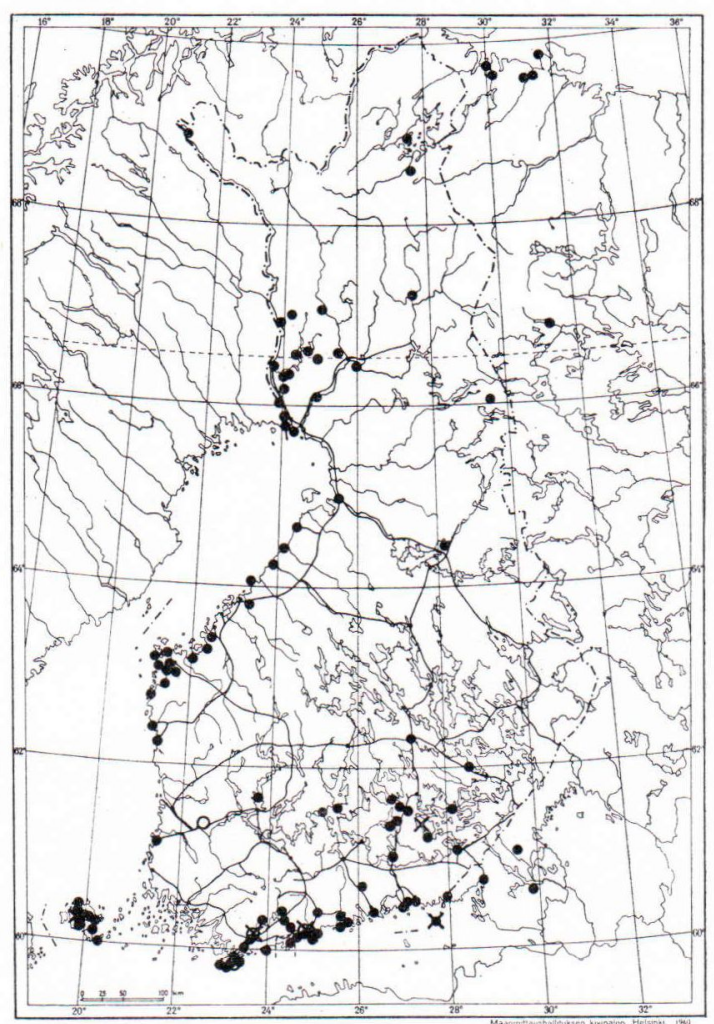

Abb. 9. Rezente Verbreitung von Diatoma elongatum (Punkte), D.e. var. actinasteroides (Ringe) und D.e. var. minor (Kreuze).

\section{Var. actinasteroides Krieger}

Eine im Plankton spärlich angetroffene Variation kühlen, schwach salzigen Wassers. Unter anderem vor Helsinki und Hanko, in der PojoBucht sowie in Säkylä gefunden.

Var. bybrida (Grun.) A. Cl.

Synonym: Diatoma tenue var. bybrida Grun.

Eine nur vor Helsinki gefundene Variation (Välikangas 1926).

Var. minor Grun.

Eine der var. tenuis stark ähnelnde Variation. Sie ist u.a. in der Pojo-Bucht und auf Åland angetroffen worden. Abb. 9.

\section{Var. pachycephala (Grun.) Hustedt}

Synonym: Diatoma tenue var. pachycephala Grun. 


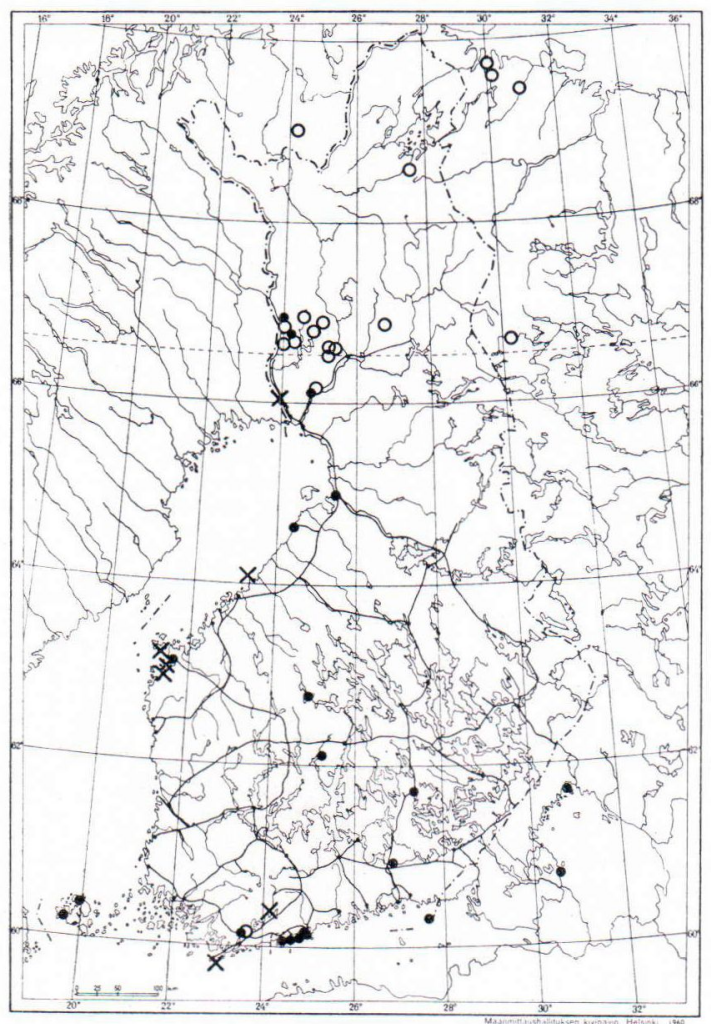

Abb. 10. Rezente Verbreitung von Diatoma elongatum var. pachycephala (Kreuze), D.e. var. tenuis (Punkte) und Diatoma biemale (Ringe).

Seltene Variation, die neben der Hauptform häufiger in brackigem als in süssem Wasser wachst. Abb. 10.

Var. subsalsa A. Cleve

Nur vor Helsinki gefundene Variation (Välikangas 1926).

\section{Var. tenuis (Agardh) van Heurck}

Synonym: Diatoma tenue Agardh.

Eine neben der Hauptform verhältnismässig allgemein vorkommende Variation. Benthosform der Litoralzone. Abb. 10. Im Balatonsee bildet sie im Frühjahr Massenvorkommen (Tamas und Gellert 1958).

Diatoma biemale (Lyngb.) Heiberg

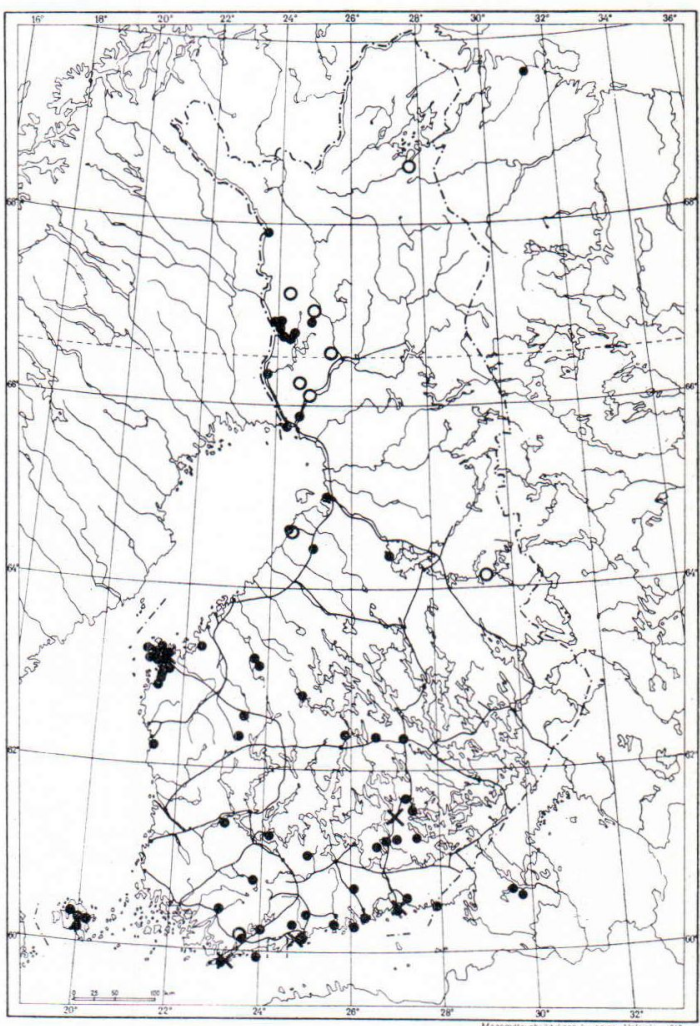

Abb. 11. Rezente Verbreitung von Diatoma biemale var. mesodon (Ringe), Diatoma vulgare (Punkte) und D.v. var. constricta (Kreuze).

Vorzugsweise in fliessenden Gewässern Lapplands wachsende Kaltwasserform. Abb. 10. Auch in den Alpen häufig (Hustedt 1931). Wahrscheinlich wächst die Art häufig im beginnenden Frühjahr auch in Mittel- und Südfinnland (das bearbeitete Material ist hauptsächlich im Frühsommer - Herbst gesammelt worden). Nach Scheele (1952) ist die Art alkaliphil.

Var. mesodon (Ehr.) Grunow

Synonyme: Fragilaria mesodon Ehr., Odontidium mesodon Kütz., Diatoma vulgare f. cuneata, f. quadrata Rabenh., Diatoma biemale var. mesodon f. rotundata Mayer.

Sehr häufige Kaltwasserform, oft häufiger als die Hauptform. Erscheint zeitweise in Bächen und Strömen massenhaft in Kolonien mit der Hauptform und Meridion circulare. Abb. 11. 


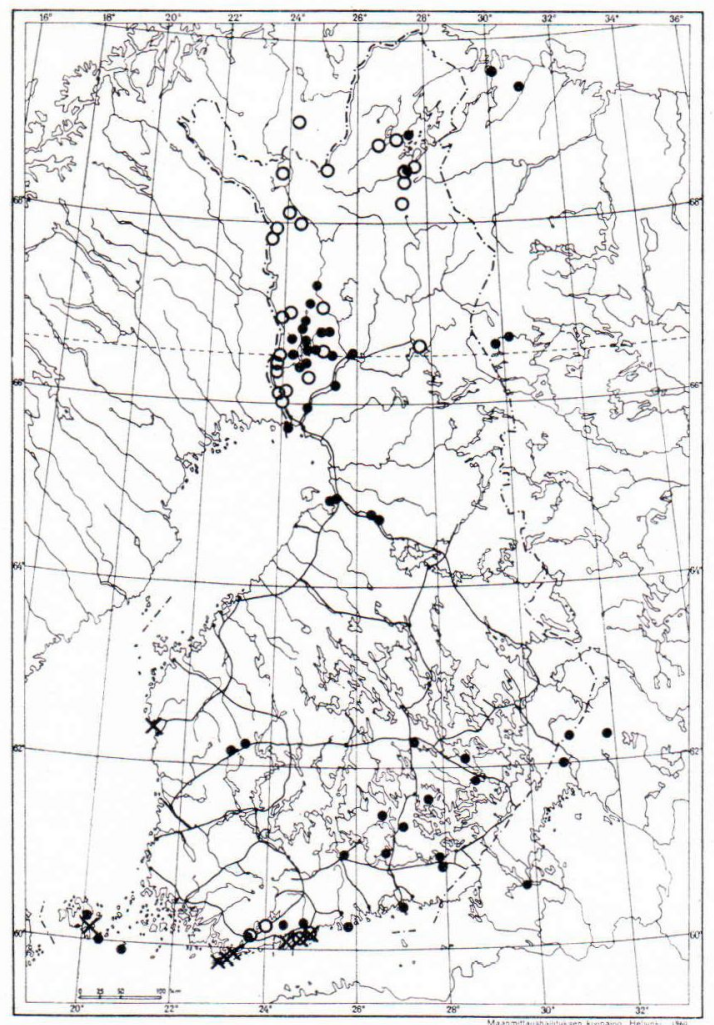

Abb. 12. Rezente Verbreitung von Diatoma vulgare var. ovalis (Ringe), D.v. var. producta (Kreuze) und Opephora martyi (Punkte).

\section{Diatoma vulgare Bory}

Alkaliphile Form, die auch in schwach salzhaltigem, kühlem Wasser der Küste wächst (Kolbe \& Tiegs, 1929: mesohalob). Abb. 11. Nach Niessen (1956) ist die Art vom Eisengehalt des Wassers in höherem Masse abhängig als vom $\mathrm{pH}$. Sie wächst in Wasser mit einem Fe- Gehalt von 2.5-5 mg/l. Nach Uspenski (1927) ist Fe eine Voraussetzung für das Wachsen der Art. Var. brevis Grunow

Eine neben der Hauptform nur selten angetroffene Variation.

Fundorte: Kaskinen, Pilgujoki in Petsamo.

\section{Var. capitulata Grunow}

Seltene Variation, die rezent nur im See Hal- suanjärvi und in der Pojo-Bucht aufgefunden worden ist.

\section{Var. constricta Grunow}

Vorwiegend eine Planktonart des Küstengebiets mit schwach brackigem Wasser. Abb. 11 . Auch kommt sie selten in marinen Sedimenten vor.

Var. linearis Grun.

Eine nur im Flusse Pahtajoki gefundene Form (Round 1959).

Var. ovalis (Fricke) Hustedt

Synonym: Diatoma ovalis Fricke.

Eine der var. brevis ähnliche Form, aber häufiger. Unter anderem in der Pojo-Bucht angetroffen. Abb. 12.

Var. producta Grunow

Nur wenig von der Hauptform unterschiedene Variation, die u.a. auf Raippaluoto und in Tvärminne festgestellt worden ist. Abb. 12.

\section{Plagiogramma}

Die Valvarebene der hier zu besprechenden Art ist länglich oval. Die Membran ist in ihrem mittleren Teil und an ihren Enden ohne Oberflächenfigurierung, aber der Zwischenteil umfasst eine durch transapikale und apikale Linien zustande gekommene areolierte Fläche. Pseudoraphe schmal. In der Mitte der Valvarfläche der Zelle liegt quer eine ovale Öffnungsfigur.

\section{Plagiogramma staurophorum (Greg.) Heiberg}

Eine maritime Form, die nach Hustedt (1931) im Küstengebiet Europas vom Mittelmeer bis zum arktischen Raum vorkommt. Doch ist die Art in der Ostsee nicht rezent, wenn auch immerhin in den oberflächlichen Sedimenten des Grundes festgestellt worden (Mölder 1962). Subfossil hat man sie in einem vom interglazialen Meere abgesetzten Tonklumpen aus Rouhiala erkannt (Brander 1941). 


\section{Dimerogramma}

Die betreffenden Arten sind klein, in ihrer Valvarfläche rhombisch länglich. Pseudoraphe verhältnismässig breit im mittleren Teil, die apikalen Enden hyalin sowie die Membran durch transapikale Punktlinien areoliert. An der Gürtelbandseite sind pervalvare Einschnürungen dicht bei den Polen zu sehen.

Dimerogramma minor (Greg.) Ralfs und var. nana (Greg.) V. Heurck

Marine Litoralformen vom Mittelmeer bis Nordeuropa (Hustedt op. cit.). Erstere selten im Ostseegrund angetroffen (Mölder 1962), beide in Interglazialsedimenten von Rouhiala (Brander 1941).

\section{Opephora}

Die Valvarfläche oval kegelförmig. Die Transapikalstreichen besonders dick (O. martyi) oder durchschnittlich dick und kurz (O. marina). Die Pseudoraphe deutlich $\mathrm{zu}$ unterscheiden. Die Gürtelbandseite schwach kegel- oder trapezförm- ig. Die Zellen wachsen, während sie von ihrem unteren Ende aus durch Gallertstränge an der Unterlage befestigt sind.

\section{Opephora martyi Héribaud}

Synonyme: Opephora martyi var. robusta Hérib., Opephora martyi f. anomala Hérib., Fragilaria mutabulis var. subsolitaris Grun., Fragilaria pinnata f. subsolitaris (Grun.) Mayer.

Eine relativ häufige Form in Seen, Flüssen sowie im Küstengebiet mit schwach brackigem Wasser. Abb. 12. Auch in Süss- wie auch Brackwassersedimenten häufig.

Opephora marina (Greg.) Pétit

Synonym: Meridion marinum Gregory.

Marine Kieselalge des Küstengebiets. Die Angaben über das Auftreten im Ostseegebiet beschränken sich auf nur eine rezente Beobachtung von Lehtisaari (Mölder und Tynni 1966), von der Oberfläche des Ostseegrunds (Mölder 1962) sowie aus interglazialen Meersedimenten von Rouhiala. Aus Rouhiala hat Brander auch Opephora pacifica dargestellt. 
TAFEL I

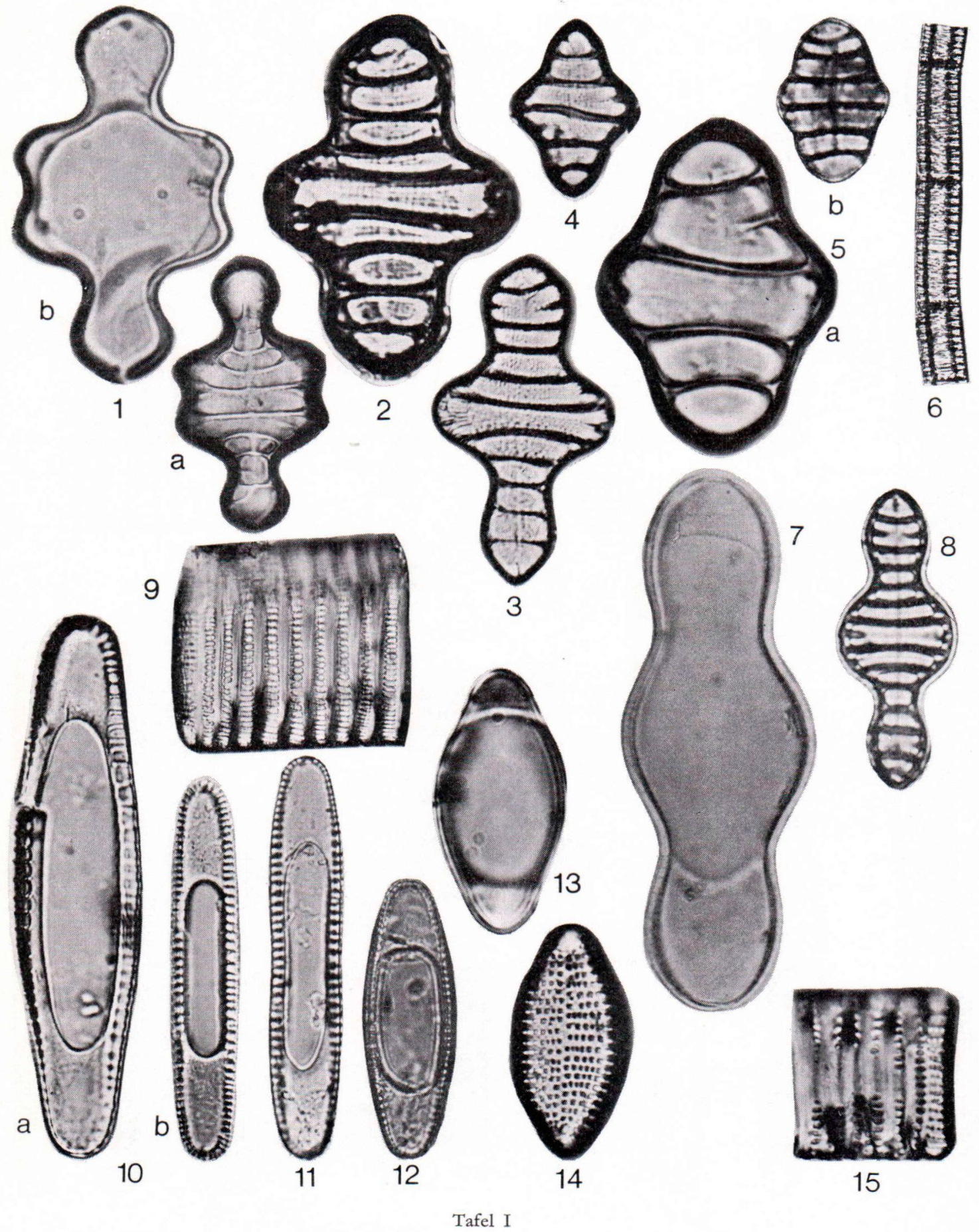

Fig. 1. a-b. Tetracyclus emarginatus, b. Zwischenband, 2. T. lacustris, 3. T. l. var. capitata, 4. T. l. var. elegans, 5. a-b

T. l. var. strumosa, 6. T. l., Kolonie, 7. T. l. fo. maxima, 8. T. l. var. capitata, Übergangsform nach T. l. fo. maxima, 9. Rhadonema arcuatum, Gürtelbandseite, 10. a-b. R. a., Schalen, 11. R. a. fo. contracta, 12. R. a., 13, 14. R. minutum, 13. Zwischenband. 15. R. m., Gürtelbandseite. 
TAFEL II

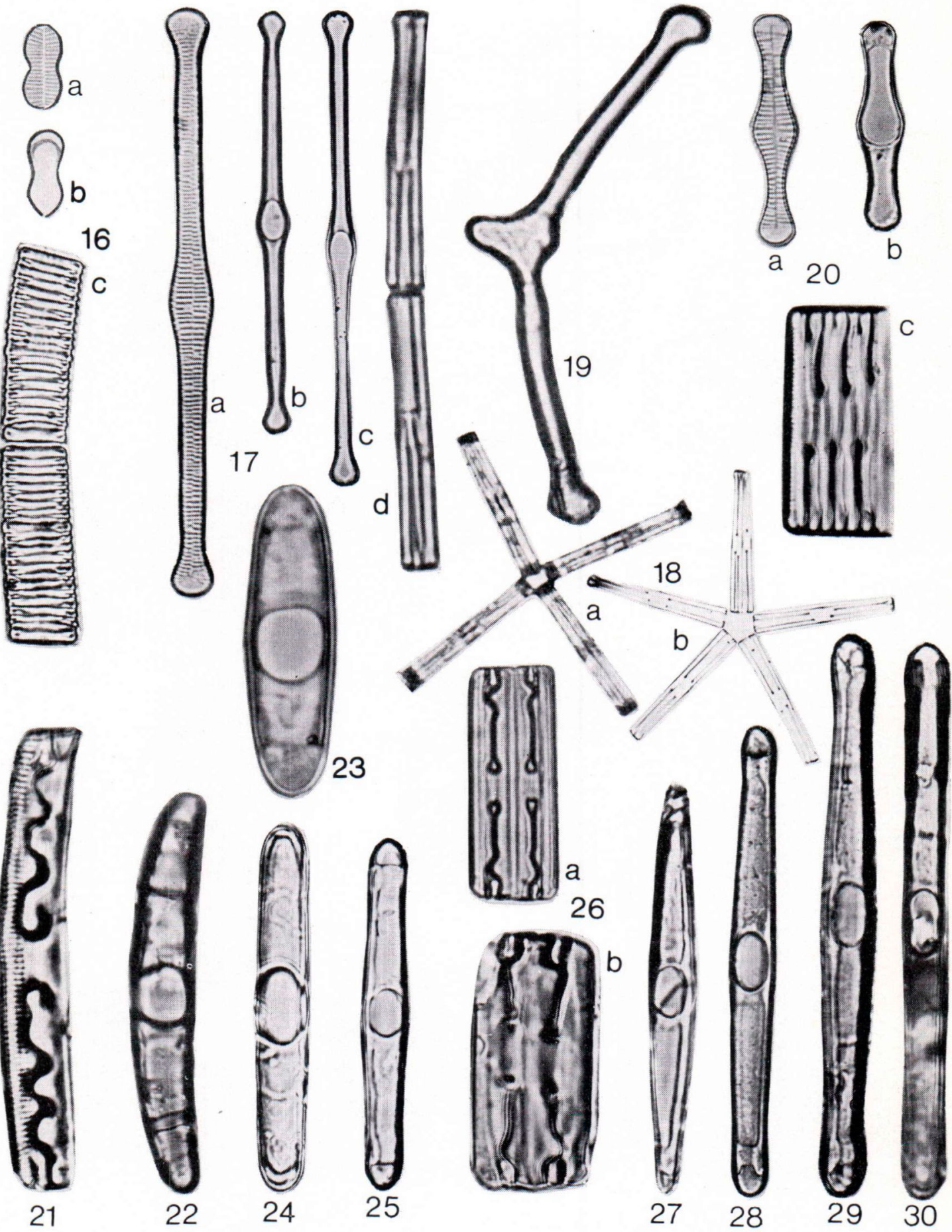

Tafel II

Fig. 16. a-c. Tabellaria binalis, a. Schale, b. Zwischenband, c. Kolonie, 17. a-d. T. fenestrata, d. Kolonie, 18. a-b. T.f. var. asterionelloides, Kolonien, 19. T. f. var. geniculata, 20. a-c. T. flocculosa, a. Schale, b. Zwischenband, c. Gürtelbandseite, 21. Grammatophora angulosa, Gürtelbandseite, 22. G. arcuata, 23, 25. G. marina, 24. G. m. var. adriatica, 26. a-b. G. m., Gürtelbandseite, 27, 28. G. oceanica, 29, 30. G. o. var. macilenta. 

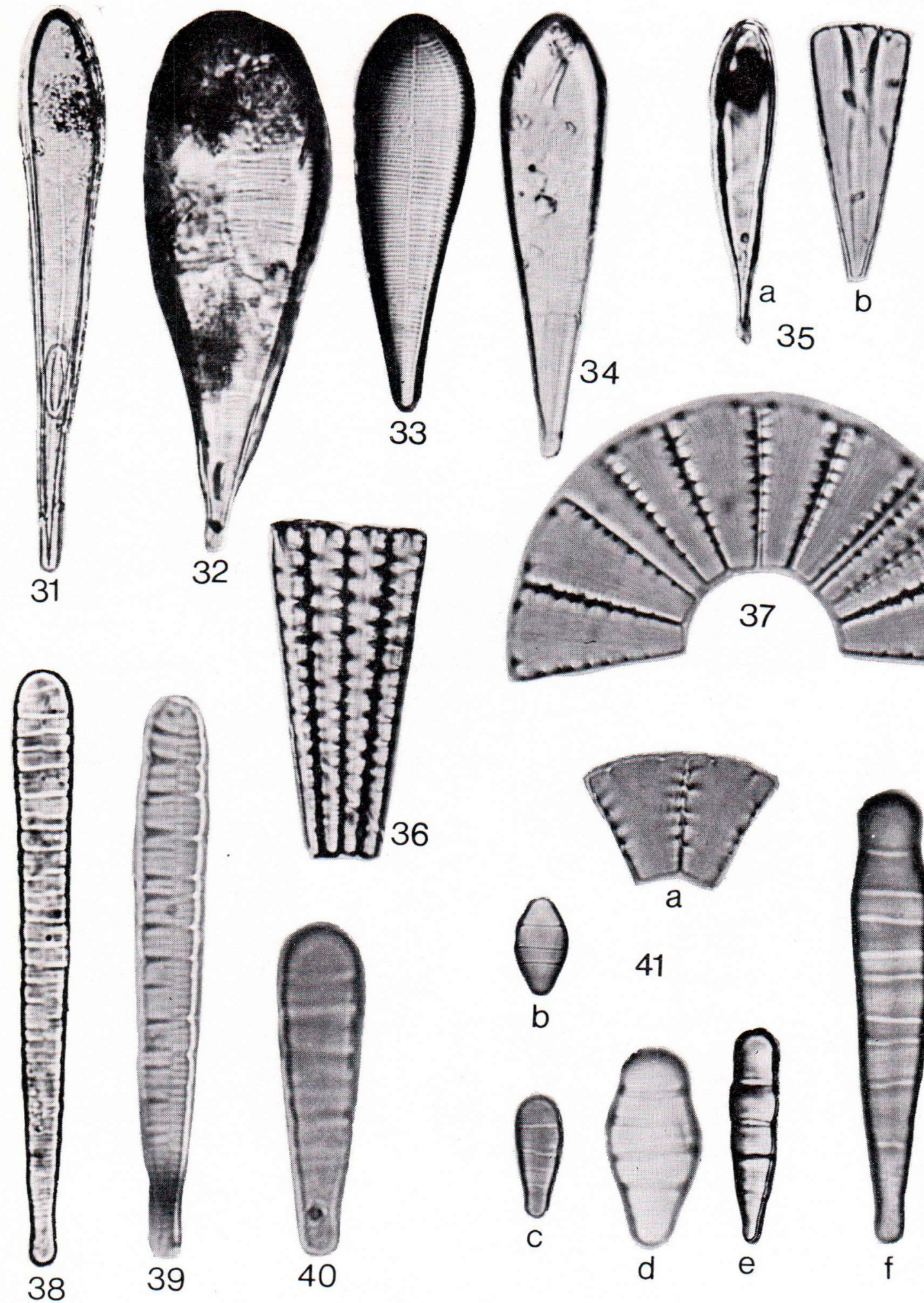

a
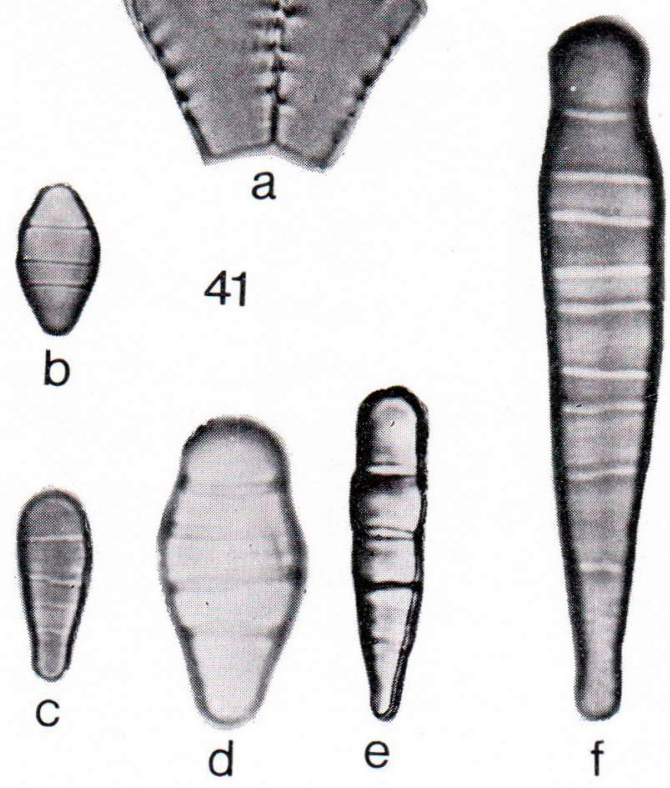

Tafel III

Fig. 31. Licmophora ebrenbergii, 32. L. e. fo. elegans, 33. L. gracilis, 34. L. nubecula, 35. a. L. paradoxa, 35. b. L. sp., Gürtelbandseite, 36, 37. Meridion circulare, Kolonien, 38-40. M. c., 41. a-f. M. c. var. constricta, a. Gürtelbandseite. 
TAFEL IV
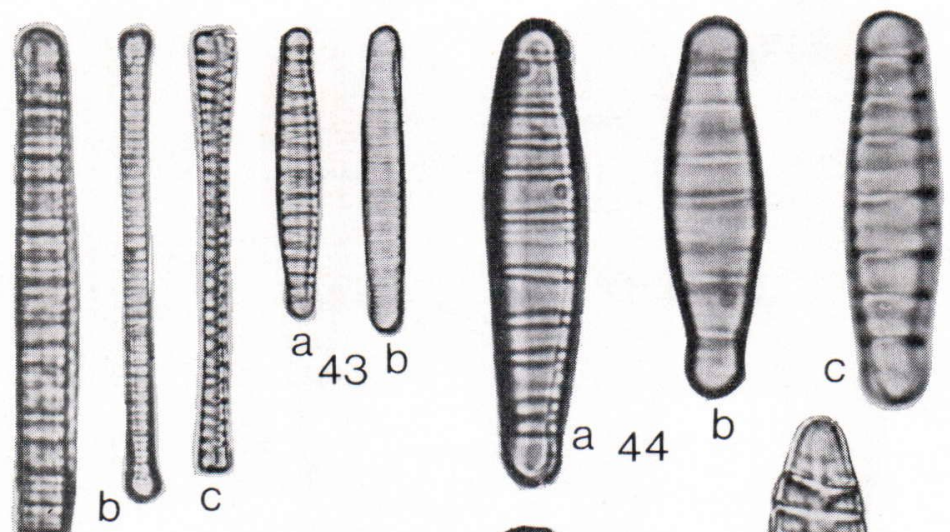

a

42
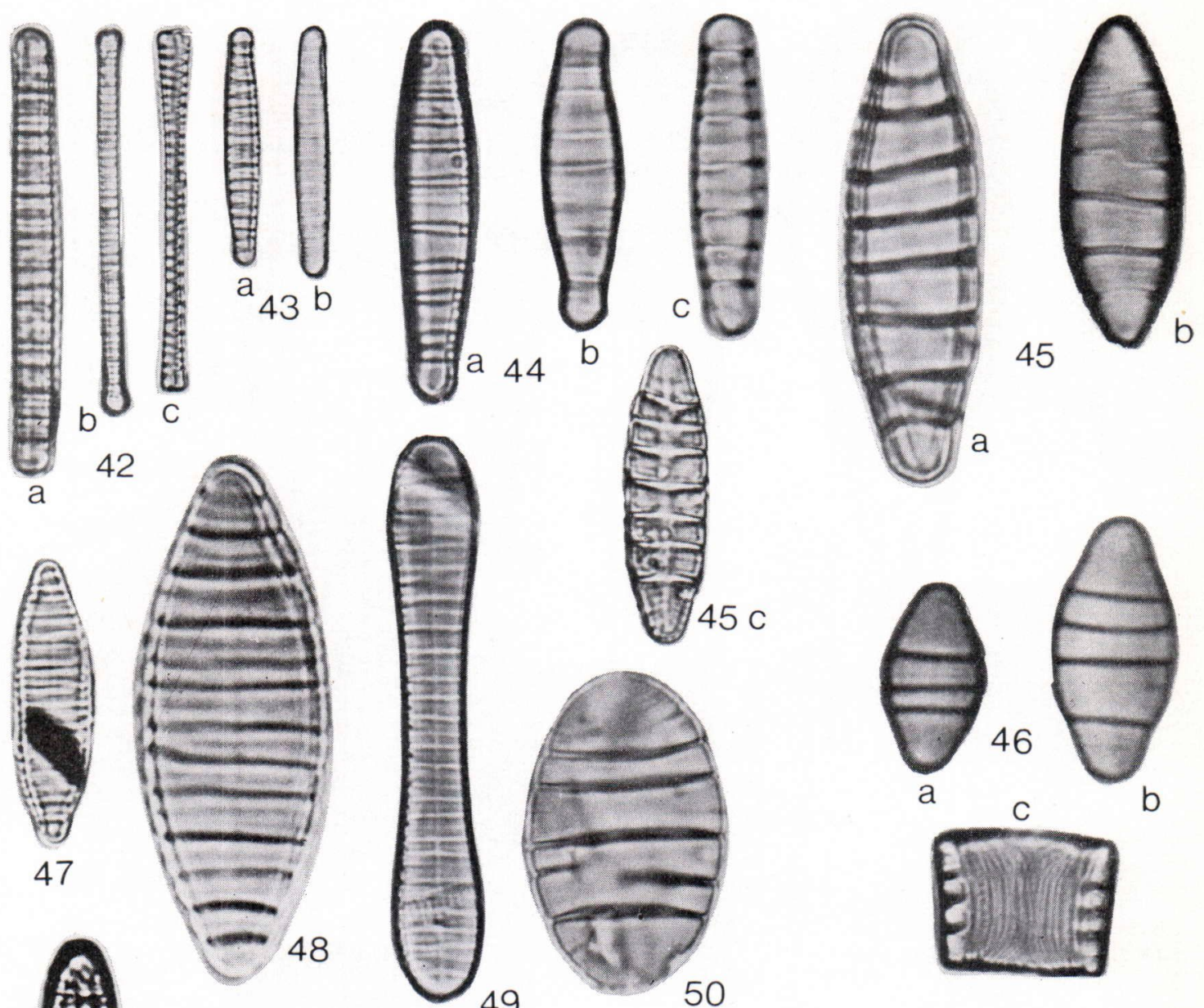

49
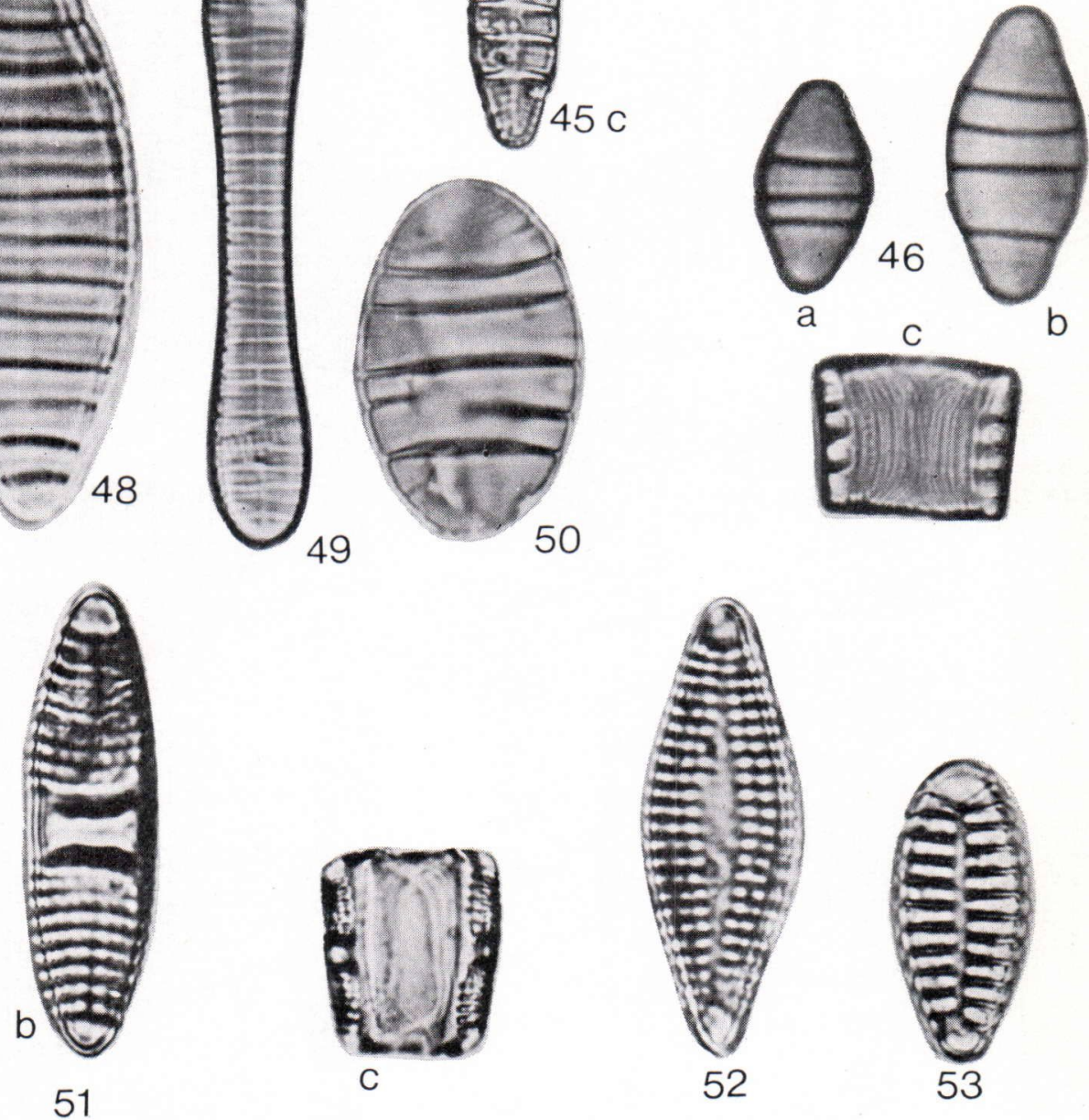

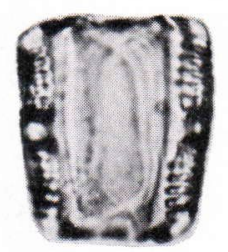

C

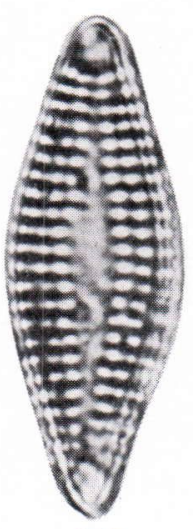

52

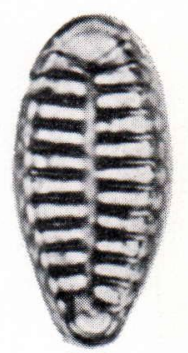

53

Tafel IV

Fig. 42. a-c. Diatoma elongatum, 43. D. e. var. tenuis, 44. a-c., 45. a-c. Diatoma biemale, 46. a-c. D. h. var. mesodon, c. Gürtelbandseite, 47. D. vulgare, 48. D. v. var. brevis, 49. D. v. var. constricta, 50. D. v. var. ovalis, 51. a-c. Plagiogramma staurophorum, 52. Dimerogramma minor, 53. Opephora martyi.

Meistens etwa 1000 -fache Vergrösserung. Photo E. Halme. 


\section{ERGÄNZENDE SCHRIFTEN}

FogED, N. (1964) Freshwater Diatoms from Spitzbergen. Tromsö Museums Skrifter Vol. 11.

Hustedt, Fr. (1931) Die Kieselalgen Deutschlands, Österreichs und der Schweiz. 2. Teil, Lif. 1. Rabenhorst: Kryptogamenflora, 7. Leipzig.

- (1942) Diatomeen aus der Umgebung von Abisko in Schwedisch-Lappland. Archiv für Hydrobiologie. Bd. 39, S. 82-174.

- (1938/1939) Systematische und ökologische Untersuchungen über die Diatomeen-Flora von Java, Bali und Sumatra. Archiv für Hydrobiologie Suppl.-Bd. 15,16 .

- (1967) Die Diatomeen des Flusssystems der Weser im Gebiet der Hansestadt Bremen. Abh. naturw. Ver. Bremen, Bd. 34, Hf. 3, S. $181-440$.

Järnefelt, H. (1935) Die Regionale Verteilung der Gewässertypen in Finnland. Verh. int. Ver.f.theor. u.angew. Limnologie. Bd. 7.

- (1956) Zur Limnologie einiger Gewässer Finnlands 17. Ann. Zool. Soc. "Vanamo» 18, 2.

Jörgensen, E. G. (1948) Diatom communities in some Danish lakes and ponds. D. Kgl. Danske Vid. Selsk. Biol. Skr. Bd. 5, Nr. 2.

Knutson, Brenda (1952) The Diatom genus Tabellaria. I. Taxonomy and Morphology. Annals of Botany, N. S. Vol. 16, No. 63.

- (1953) The Diatom Genus Tabellaria. II. Taxonomy and Morphology of the Plankton Varietes. Ann. of. Bot., N. S. Vol. 17, No. 65.

- (1954) The Ecology of the Diatom genus Tabellaria in the English lake district. Journal of Ecology, Vol. $42,2$.

Kolbe, R. W. und A. W. Silfyersparre (1950) Úber ein Massenvorkommen und weitere rezente Standorte der Kieselalge Tabellaria binalis (Ehr.) Grun. in Schweden. Svensk Bot. Tidskr. 44, 1.

Kolbe, R. W. und Tiegs, E. (1929). Zur mesohaloben Diatomeenflora des Werra gebiets. Ber. deutsch. bot. Ges., Bd. 47.

Krasske, G. (1938) Beiträge zur Kenntnis der DiatomeeVegetation von Island und Spitzbergen. Archiv, für Hydrobiol. Bd. 33, S. 503-533.
Levander, K. M. und Y. Vuorentaus (1915) III. Planktonsammansättningen i Kemi, Uleå och Kumo älf samt Kymmene och Saima system på grund af från juni 1913 till juni 1914 månatligen utförda håfningar. Fennia 39, no 2 .

- (1917) IV. Planktonsammansättningen i Finska insjöar och floder på grund af nåfningar utförda sommaren 1913. Fennia 40, no 6.

Miller, Urve (1964) Diatom floras in the Quaternary of the Göta river valley (Western Sweden). Sveriges Geol. Unders. Ser. Ca, Nr 44.

Mölder, K. (1951) Die Diatomeenflora einiger Eisrandstandorte in Norwegen und Island. Arch. Soc. Zool. Bot. Fennicae "Vanamo» 5: 2.

Niessen, Herta (1956) Ökologische Untersuchungen über die Diatomeen und Desmidiaceen des Murnauer Moores. Arch. f. Hydrobiol. 51, 3, S. 281-375.

QuenNerstedt, N. (1949) Om diatoméerna Actinella punctata Lewis och Tabellaria binalis (Ehr.) Grun. i Svenska vatten. Svensk Bot. Tidskr. Bd. 43, H. 1.

Round, F. E. (1960) The epipelic algal Flora of some Finnish lakes. Arch. Hydrobiol. 57, S. 161-178.

SCHEELE, M. (1952) Systematisch-ökologische Untersuchungen über die Diatomeenflora der Fulda. Archiv. f. Hydrobiol. Bd. 46, S. 305-423.

Sснмidt, A. (1873-1944) Atlas der Diatomaceenkunde. Aschersleben u. Leipzig.

Tamas, Gizella u. Gellért, J. (1958) Detritusz-turzások kovamoszatainak és csillósainak ökológiai vizsgálata a Tihanyi-félsziget keleti partjèn. Referat: Ökologische Untersuchungen an Diatomeen und Ciliaten der Detritus-Drifte am Ostufer der Halbinsel Tihany. Annal. Biol. Tihany.

Uspenski, E. E. (1927) Eisen als Faktor für die Verbreitung niederer Wasserorganismen. Planzenforschung H. 9.

Zans, V. (1936) Das letzinterglaziale Portlandia-Meer des Baltikums. Suomen Geologinen Seura n:o 9, Bull. Comm. géol. Finlande 115. S. 231-250.

Manuskript eingegangen am 31. Januar 1969

Anschrift: Prof. Karl Mölder, Kelohongantie 2 C 21 Tapiola, Finnland 